\title{
Chemical Reaction Optimization for Feature Combination in Bio-inspired Visual Attention*
}

\author{
Lu Gan \\ State Key Laboratory of Virtual Reality Technology and Systems, School of Automation Science and Electrical \\ Engineering, Beihang University \\ Beijing, 100191, PR China ${ }^{\dagger}$ \\ Haibin Duan ${ }^{1,2}$ \$ \\ 1.State Key Laboratory of Virtual Reality Technology and Systems, School of Automation Science and Electrical \\ Engineering, Beihang University \\ 2. Science and Technology on Aircraft Control Laboratory, Beihang University \\ Beijing, 100191, PR China
}

Received 17 May 2013

Accepted 9 February 2015

\begin{abstract}
Bio-inspired visual attention models human visual system to detect the most salient part of a visual field. In the existing diversified computational models, bottom-up visual attention that works out a saliency map to indicate the conspicuity of visual stimuli in an image has gained much popularity. This paper introduces a task-driven training procedure into the basic bottom-up computational model to make bio-inspired visual attention more intelligent and appropriate for a particular visual task. Chemical Reaction Optimization (CRO) is a recently proposed evolutionary metaheuristic, simulating the dynamic interaction of molecules in a chemical reaction. In this paper, CRO algorithm is used to optimize the weight coefficients for feature combination through the training procedure. Experimental results show that $\mathrm{CRO}$ algorithm outperforms other evolution algorithms in bio-inspired visual attention.
\end{abstract}

Keywords: bio-inspired visual attention, Chemical Reaction Optimization (CRO), feature combination, saliency

*Corresponding Author: Haibin Duan, Email: hbduan@buaa.edu.cn. 


\section{Introduction}

Data processing in real-time is quite a difficult task for today's machine vision, especially when it deals with high-resolution images. However, human visual system can process a rich stream of visual data very efficiently by focusing on the salient part ${ }^{1}$. Based on this mechanism, bio-inspired visual attention model has been established and widely applied in scene analysis ${ }^{2}$, visual recognition ${ }^{3}$, and object detection ${ }^{4}$ over the recent decades.

Many computer vision researchers ${ }^{2,5,6}$ psychologists $^{7,8}$ and neuroscientists ${ }^{9}, 10$ have been investigating the mechanisms of visual attention, and a wide spectrum of computational models have been proposed. The models of visual attention can be classified as bottom-up (driven by the scene) or top-down (driven by the task) models. Bottom-up model is usually a feedforward network which is efficient but sometimes invalid ${ }^{2}$, while top-down model a feedback loop which is effective but time-consuming ${ }^{11}$. Integration of bottom-up method and top-down method is a destination for researchers ${ }^{12,13}$. This paper introduces a task-driven training procedure into a bottom-up model to combine the conciseness and efficiency of bottom-up method with the effectiveness and flexibility of top-down method.

The training procedure is established based on a bottomup bio-inspired visual attention model ${ }^{2}$ proposed by Itti et al. This model combines intensity, color and orientation features to work out a saliency map in a purely scene-driven manner. In a specific visual task, every stimulus could be conspicuous in a saliency map because of no previous information about the target, which makes the model aimless and confusing. In this paper, we utilize Chemical Reaction Optimization (CRO) algorithm to train the weight coefficients which are used to combine several feature maps. Through an optimizing phase, the weight coefficients are more appropriate for a specific visual target in feature combination stage.

$\mathrm{CRO}$ is a recently developed optimization algorithm proposed by Lam and $\mathrm{Li}$, which mimics the transition and interaction of molecules in a chemical reaction ${ }^{14}$. Since CRO algorithm was established, a lot of work about it has been done and demonstrated its superior optimization performances $^{15-18}$. In this paper, CRO algorithm is employed to overcome the aimlessness of feature combination in Itti's visual attention model.
After the optimization of feature combination process by CRO algorithm, we found that our visual target is much more conspicuous than it is in the basic model. Three groups of comparative experiments have been carried out in this paper and demonstrated the effectiveness and efficiency of our method.

The remainder of this paper is organized as follows. In Section 2, we briefly describe Itti's bottom-up model and our task-driven training procedure. Section 3 introduces the basic principles and operators of CRO algorithm. In Section 4, the design of our proposed CRO-based visual attention is described in detail. Section 5 provides some comparative experimental results and concluding remarks are finally given in Section 6.

\section{Joint Visual Attention}

Visual attention is a biologically inspired mechanism to reduce the amount of visual data and reserve the most salient part ${ }^{5}$, which is very useful in image processing, robot and computer vision. In recent decades, there are lots of computational models for visual attention, from bottom-up to top-down methods. Itti's model is one of the most influential bottom-up models ${ }^{2}$, which we briefly describe in this section. Then, the procedure of our weight coefficients training in a top-down manner is given.

\subsection{Basic model of bottom-up visual attention}

This bottom-up model is proposed by Itti et al. to explain human visual search strategies, based on the hierarchical biologically-plausible architecture of Koch and Ullman ${ }^{19}$. The first stage of this model is decomposing the input image into three separate feature channels (i.e., color, intensity and orientation) to extract early visual features. The feature maps of each channel are computed in parallel.

Each channel creates its own pyramid by increasingly low-pass filtering and subsampling the input image. In the intensity channel, an intensity image $I$ can be obtained by Eq. (1).

$$
I=\frac{1}{3}(r+g+b)
$$

where $r, g$ and $b$ are respectively the red, green and blue channel of the input image. In the color channel, four color images $R, G, B$ and $Y$ are calculated with Eq. (2): 


$$
\left\{\begin{array}{l}
R=r-\frac{1}{2}(g+b) \\
G=g-\frac{1}{2}(r+b) \\
B=b-\frac{1}{2}(r+g) \\
Y=\frac{1}{2}(r+g)-\frac{1}{2}|r-g|-b
\end{array}\right.
$$

where $R, G, B$ and $Y$ represent red, green, blue and yellow image, respectively. In the orientation channel, orientation images $O(\theta)$ are obtained from the intensity image $I$ using Gabor filters with different preferred orientations $\theta \in\left\{0, \frac{1}{4} \pi, \frac{1}{2} \pi, \frac{3}{4} \pi\right\}$. Then, the Gaussian pyramid with nine spatial scales of each image is created for the calculation in the next stage.

The second stage contains center-surround differences and normalization of output pyramids from the first stage. The center-surround difference operator is denoted by $\Theta$, representing across-scale difference between two images with different scale level in previous pyramids. Since the scale level of the center is defined as $c \in\{2,3,4\}$ and that of the surround as $s=c+o$, where $o \in\{3,4\}$, we can implement centersurround differences as follows:

$$
\left\{\begin{array}{l}
I(c, s)=|I(c) \Theta I(s)| \\
R G(c, s)=|(R(c)-G(c)) \Theta(\mathrm{G}(s)-R(\mathrm{~s}))| \\
B Y(c, s)=|(B(c)-Y(c)) \Theta(Y(s)-B(\mathrm{~s}))| \\
O(c, s)=|O(c) \Theta O(s)|
\end{array} .\right.
$$

Therefore, through the second stage 42 feature maps including 6 intensity maps, 12 color maps and 24 orientation maps are obtained.

In the third stage, feature maps are across-scale combined and normalized into three conspicuity maps using Eq. (4):

$$
\left\{\begin{array}{l}
\bar{I}=\bigoplus_{c=2}^{4} \bigoplus_{s=c+3}^{c+4} N(I(c, s)) \\
\bar{C}=\bigoplus_{c=2}^{4} \bigoplus_{s=c+3}^{c+4}[N(R G(c, s)+N(B Y(c, s))], \\
\bar{O}=\sum_{\theta \in\left\{0, \frac{1}{4} \pi, \frac{1}{2} \pi, \frac{3}{4} \pi\right\}} N\left(\bigoplus_{c=2}^{4} \bigoplus_{s=c+3}^{c+4} N(O(c, s, \theta))\right)
\end{array}\right.
$$

where $N($.$) is a map normalization operator, \oplus$ denotes across-scale addition, $\bar{I}, \bar{C}$ and $\bar{O}$ are the conspicuity maps computed in the three channels, respectively.
Finally, three conspicuity maps are linearly combined into a single saliency map which indicates the conspicuousness of the input image at each pixel:

$$
S=\frac{1}{3}(N(\bar{I})+N(\bar{C})+N(\bar{O})) .
$$

However, there are two problems in such feature combination strategy. One is that since different types of features are incomparable with each other, averaging them is meaningless. The other one is that image noise could be superimposed due to the simple integration of many feature maps. To solve these problems, we introduce a top-down training procedure into the basic bottom-up model to optimize the feature combination process for a specific visual task.

\subsection{The task-driven training procedure}

For a specific visual task, each feature map obtained in the second stage of this model must correspond to an optimal weight coefficient to be integrated into the final saliency map. Therefore, a training procedure is employed to search the most suitable weight coefficients for a certain visual target. We improved the last two stages with the following equation:

$$
S=\frac{1}{\sum_{i=1}^{6} W_{1 i}+\sum_{j=1}^{12} W_{2 j}+\sum_{k=1}^{24} W_{3 k}}\left(\sum_{i=1}^{6} W_{1 i} \times I(i)+\sum_{j=1}^{12} W_{2 j} \times C(j)+\sum_{k=1}^{24} W_{3 k} \times O(k)\right),
$$

where $I(i), C(j)$, and $O(k)$ represent the feature maps obtained in the intensity, color, and orientation channel, respectively, $W_{1 i}(i \in\{1,2, \ldots, 6\})$, $W_{2 j}(j \in\{1,2, \ldots, 12\})$ and $W_{3 k}(k \in\{1,2, \ldots, 24\})$ are the 42 weight coefficients to be optimized.

In the training procedure, we use several training images which contain the same target to search the optimal weight coefficients by CRO algorithm. Since each training image corresponds to a set of coefficients, the optimal set of weight coefficients is obtained by a linear combination and normalization of all sets of them Then, the optimal weight coefficients are used to combine feature maps of a test image. The architecture of our joint visual attention model is presented in Fig. 1. 


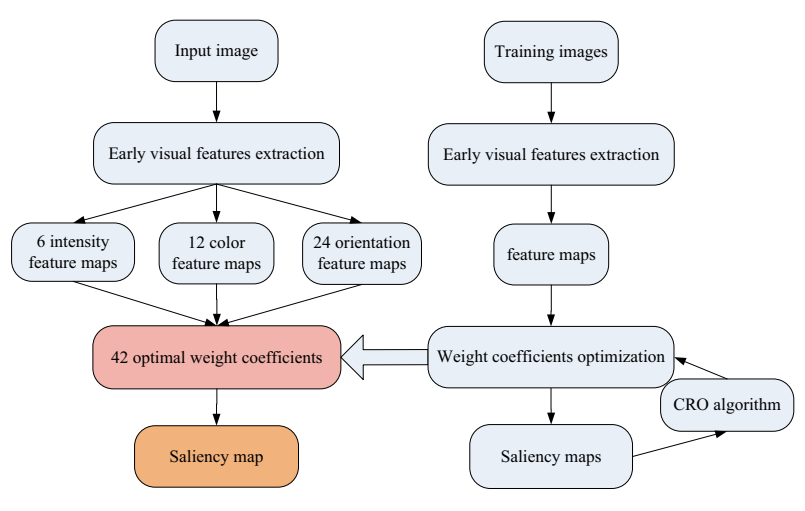

Fig. 1. Description of our joint visual attention model.

\section{Chemical Reaction Optimization}

\subsection{Principles of CRO algorithm}

CRO is a recently developed heuristic algorithm for optimization, inspired by the molecular behaviors in a chemical reaction ${ }^{20}$. Unlike other population-based metaheuristics, e.g., Artificial Bee Colony $^{21}$, Differential Search ${ }^{22}$, Particle Swarm Optimization $(\mathrm{PSO})^{23}$ and Biogeography-Based Optimization $(\mathrm{BBO})^{24}$, the size of population in CRO algorithm is not a constant in iterative process. CRO simulates the whole process of a chemical reaction where molecules collide with the walls of a container (i.e., on-wall ineffective reaction and decomposition reaction) and with each other (i.e., inter-molecular reaction and synthesis reaction).

In $\mathrm{CRO}$ algorithm, a molecule is the basic unit with several properties such as structure $\omega$, potential energy $P$, kinetic energy $K$ and some other user-defined properties for a particular problem, where $\omega$ represents a solution of the problem, $P$ is the fitness value and $K$ quantifies the capability of a molecule to escape from a local optimum ${ }^{20}$. In a chemical reaction, the initial reactants with excessive potential energy are finally turned into products with minimal potential energy through a series of interactions.

As stated above, four types of elementary reactions are defined in CRO algorithm. An on-wall ineffective reaction occurs when a single molecule hits a wall and rebounds, i.e., $\omega \rightarrow \omega^{\prime}$. In inter-molecular ineffective reaction, two molecules collide with and then bounce off each other, i.e., $\omega_{1}+\omega_{2} \rightarrow \omega_{1}^{\prime}+\omega_{2}^{\prime}$. Decomposition reaction refers to the situation in which a molecule hits a wall and breaks into two separate parts, i.e., $\omega \rightarrow \omega_{1}^{\prime}+\omega^{\prime}{ }_{2}$, whereas synthesis reaction is a contrary situation in which two molecules collide with each other and are integrated into one molecule, i.e., $\omega_{1}+\omega_{2} \rightarrow \omega^{\prime}$. In $\mathrm{CRO}$ algorithm, which type of reaction would happen in an iteration is decided by some criteria given in Ref. [20]. Besides, all reactions must comply with two fundamental assumptions:

$$
\left\{\begin{array}{l}
\sum_{i=1}^{s}\left(P\left(\omega_{i}\right)+K\left(\omega_{i}\right)+b\right)=C \\
\sum_{j=1}^{l} P\left(\omega_{j}^{\prime}\right) \leq \sum_{i=1}^{k}\left(P\left(\omega_{i}\right)+K\left(\omega_{i}\right)\right)
\end{array},\right.
$$

where $C$ is the constant total energy of the closed system, $b$ denotes the energy in the buffer, $s, l$ and $k$ represent the number of all molecules in the system, the number of molecules after a reaction and before a reaction, respectively, $P\left(\omega_{i}\right)$ and $K\left(\omega_{i}\right)$ are the potential energy and kinetic energy of molecule $i$. Only the two assumptions are satisfied can a valid reaction be carried out. Once a molecule with minimal potential energy is produced in a reaction, the optimal solution of the problem is obtained.

\subsection{Operators in CRO algorithm}

CRO algorithm uses a neighborhood search operator to find a better solution. In this paper, we choose Gaussian mutation with reflection strategy in (both on-wall and inter-molecular) ineffective reaction and decomposition reaction. The implement of this operator can be described by Eq. (8):

$$
\omega^{\prime}(i)= \begin{cases}\omega(i)+\delta_{i} & a_{i} \leq \omega(i)+\delta_{i} \leq b_{i} \\ 2 a_{i}-\left(\omega(i)+\delta_{i}\right) & \omega(i)+\delta_{i}<a_{i} \\ 2 b_{i}-\left(\omega(i)+\delta_{i}\right) & \omega(i)+\delta_{i}>b_{i}\end{cases}
$$

where $\omega(i)$ is a randomly selected element in a molecule, $\delta_{i}$ is a random number generated by a Gaussian probability density function, and $\left[a_{i}, b_{i}\right]$ is the limit of the element in a new molecule.

The output of an on-wall ineffective reaction $\omega^{\prime}$ can be directly obtained from the above equation. In an intermolecular reaction, $\omega_{1}^{\prime}$ and $\omega_{2}^{\prime}$ are firstly assigned to be the product of $\omega_{1}$ and $\omega_{2}$, or vice versa, then processed by Eq. (8) separately. In a decomposition reaction, we produce the output molecules $\omega_{1}^{\prime}$ and $\omega^{\prime}{ }_{2}$ by randomly choosing half of the elements to be processed by the operator.

In a synthesis reaction, probabilistic select method is utilized to search an adjacent better solution: 


$$
\omega_{i \in\{1,2, \ldots, n\}}^{\prime}(i)=\left\{\begin{array}{ll}
\omega_{1}(i) & r>t \\
\omega_{2}(i) & r \leq t
\end{array},\right.
$$

where $r$ is a random number, $t$ is a pre-set threshold, and $n$ is the dimension of a molecule. In this way, each element in $\omega$ is set equal to its correspondence in $\omega_{1}$ or $\omega_{2}$ depending on probability. Through these operators, four types of reactions can be successfully implemented.

\section{Visual Attention Improved by CRO algorithm}

\subsection{Optimization using CRO algorithm}

As shown in Fig. 1, the optimal weight coefficients for a specific object can be obtained by using CRO algorithm in a training process. In this process, the molecular structure $\omega(i), i \in\{1,2, \ldots, 42\}$ is defined as a set of weight coefficients for feature combination. Since CRO is designed to search for a minimum potential energy, we define the fitness value in our method as the reciprocal of Signal-to-Noise Ratio (SNR):

$$
P(\omega)=\frac{\text { Noise }}{\text { Signal }},
$$

where Signal and Noise denote the normalized saliency of the region where our target is located, and of the background in the training image, respectively, with the molecular structure $\omega$ (Fig. 2). The normalized saliency of the whole training image using the weight coefficients in $\omega$ can be obtained by Eq. (6).

When CRO algorithm finds out a minimum value of $P\left(\omega_{o}\right)$, i.e., the maximum value of SNR, the structure of molecule $\omega_{o}$ is the best solution of our problem, i.e., a set of optimized weight coefficients for our target in visual attention. Since several sets of optimized coefficients will be obtained after involving a series of training images. We define the final optimal weight coefficients by a linear combination and normalization of all sets of optimized ones. They have powerful effects on inhibiting image noise and highlighting the target. In the test procedure, these optimal weight coefficients are used to combine feature maps produced by the test image. We can obtain a saliency map where our target is much more conspicuous than in the original Itti's model.

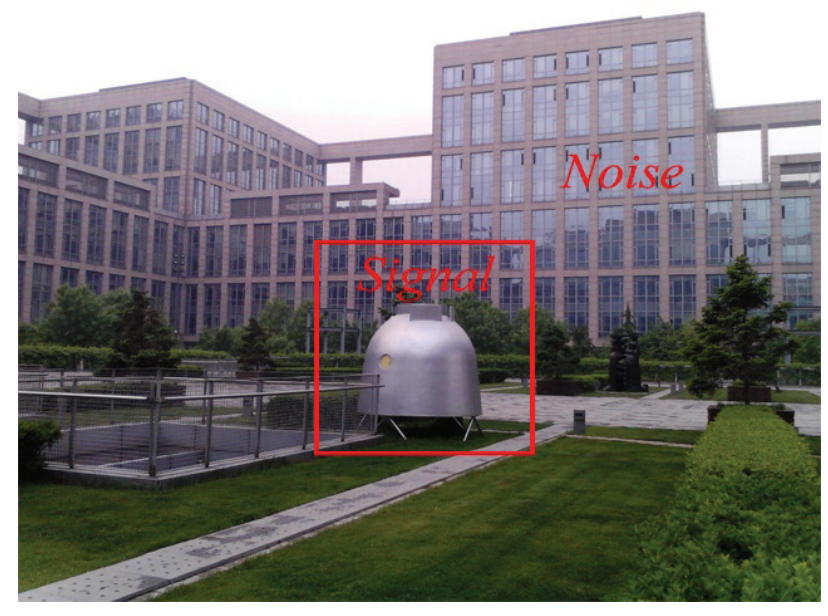

Fig. 2. Measure of Signal and Noise. In this training image, our target is inside the red rectangle and background outside the red rectangle. Signal and Noise are the normalized saliency of the region of target and background, respectively.

\subsection{Procedure of CRO-based visual attention}

Our proposed CRO-based visual attention uses a population-based algorithm $\mathrm{CRO}$ to optimize the weight coefficients for feature combination. After a task-driven training procedure, the weight coefficients are quite suitable for the target in a specific visual task. Procedure of our method is described below in detail:

Step 1: Obtain the training image and extract early visual features in intensity, color and orientation channel separately and create Gaussian pyramid for each channel (cf. Eqs. (1) and (2)).

Step 2: Compute 42 feature maps (6 intensity maps, 12 color maps and 24 orientation maps) by center-surround differences of Gaussian pyramids according to Eq. (3).

Step 3: Initialize the parameters of $\mathrm{CRO}$, including the initial state of this reaction $(s(0), K(0), b(0))$, the maximum number of iterations $M i$, two thresholds $T d$ and $T s$, which are depicted in Ref. [20].

Step 4: Randomly initialize the structure of each molecule which represents a set of weight coefficients used in feature combination stage.

Step 5: Run CRO algorithm. In each iteration, the type of reaction is chosen by the criteria described in Ref. [20]. Test whether this reaction satisfies two assumptions given in Eq. (7). If so, implement the operator stated in Section 3.2. Otherwise, cancel it immediately.

Step 6: Check for any new minimum after a reaction and reserve it. Check whether the stopping criteria are satisfied. If so, stop CRO algorithm and output the 
result, otherwise return to Step 5. After CRO algorithm completes, a set of optimized weight coefficients are obtained.

Step 7: Record all sets of optimized weight coefficients calculated from several training images and make a linear combination with them to obtain the final optimal weight coefficients. Compute a saliency map of the test image using the optimal weight coefficients according to Eq. (6).

The flow char of our training procedure with a training image using $\mathrm{CRO}$ algorithm is given in Fig. 3.

\section{Experiments and Analyses}

To verify the effectiveness and applicability of our CRO-based bio-inspired visual attention, three groups of experiments are conducted in this section. In these experiments, the parameter setting of CRO algorithm is described in Table 1.

Experiments are implemented in MATLAB 2012b on a PC with 2.9-GHz CPU, 4-GB RAM, and 32-b Windows 7. In each group of experiments, we use several training images to optimize the weight coefficients and one test image to compute a saliency map with the optimal weight coefficients. Images used in Experiment 1 and 2 are from iLab Image Databases ${ }^{25}$, and images in Experiment 3 are taken in our campus. All images are normalized in size as $640 \times 480$. Results of these experiments are shown in Figs. 4-6.

Table 1. Main parameters of CRO algorithm.

\begin{tabular}{lll}
\hline Symbol & Mathematical Meaning & Quantity \\
\hline$s(0)$ & Initial number of molecules & 10 \\
$K(0)$ & Initial kinetic energy in each molecule & 200 \\
$b(0)$ & Initial energy in the buffer & 1000 \\
$M i$ & Maximum number of iterations & 100 \\
$T d$ & Threshold defined for decomposition & 1000 \\
$T s$ & Threshold defined for synthesis & 50 \\
\hline
\end{tabular}

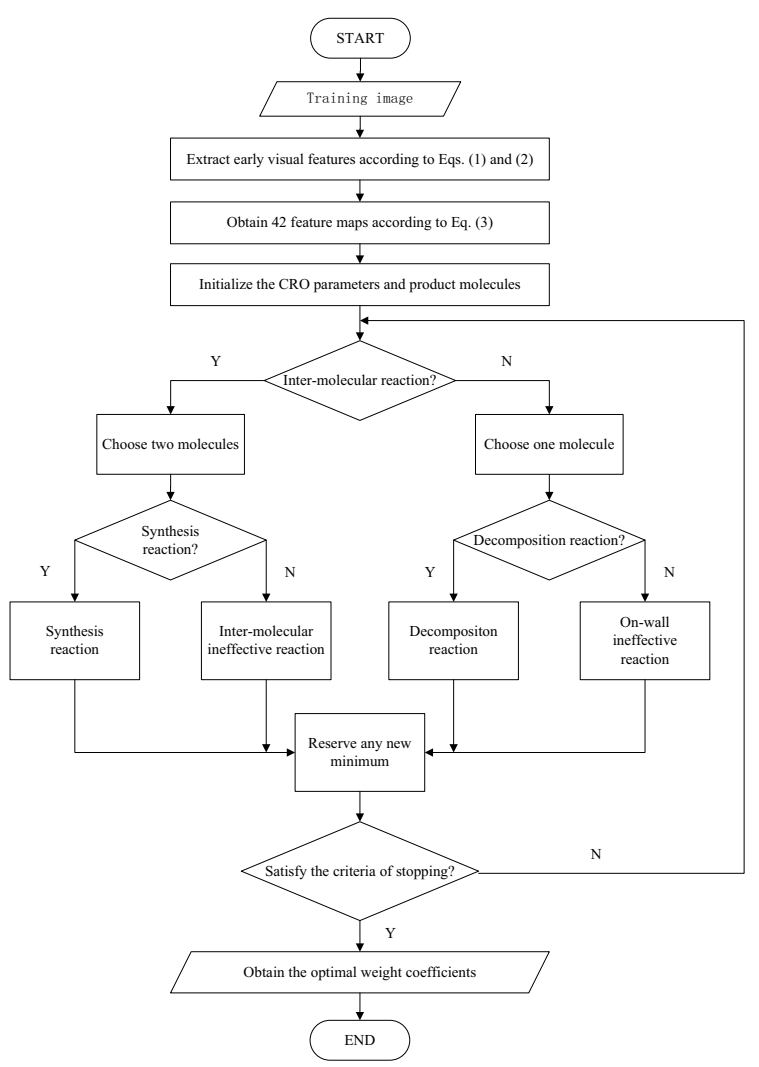

Fig. 3. Flow chart of the training procedure using CRO algorithm.

Furthermore, we compared searching performance of CRO with that of PSO and BBO by using each of them 10 times to optimize a set of weight coefficients. For each algorithm, the images, visual target, experimental platform and initial size of population are all the same. These results are given in Fig. 7.

Results show that CRO outperforms PSO and BBO in the training procedure of joint visual attention. In addition, the high accuracy and strong stability of CRO have been demonstrated in experiments. Performances of the three algorithms are analyzed in Table 2.

Since computing is consecutive in CRO but parallel in PSO and BBO, we consider $s$ reactions in CRO algorithm as an iteration for comparison, where $s$ is the

Table 2. Performances of CRO, PSO and BBO in visual attention.

\begin{tabular}{|c|c|c|c|}
\hline Algorithm & Convergence Iteration & Total Time(s) & Convergence Time(s) \\
\hline $\mathrm{CRO}$ & 15 & 50.9755 & 7.6463 \\
\hline PSO & 71 & 99.8073 & 70.8632 \\
\hline $\mathrm{BBO}$ & 58 & 102.8673 & 59.6630 \\
\hline
\end{tabular}




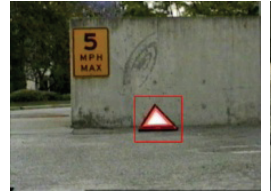

(a)

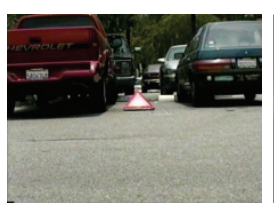

(d) (b)

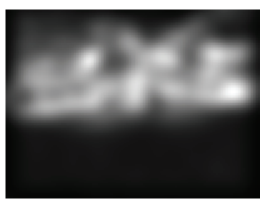

(e)

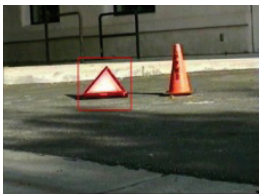

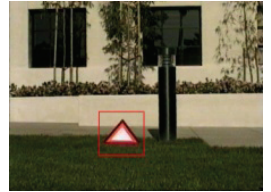

(c)

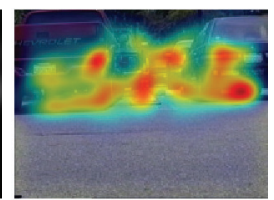

(f)

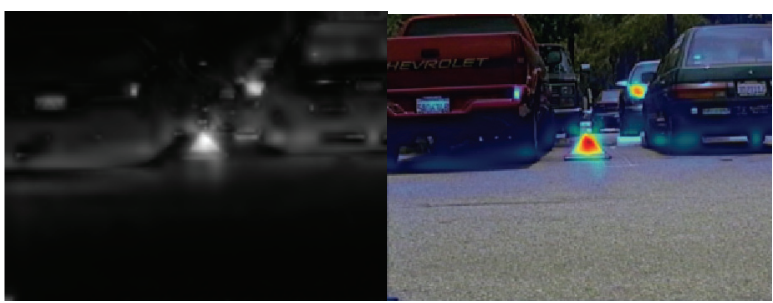

(g)

(h)

Fig. 4. Results of Experiment 1 where our target is a triangle (all images are from iLab Image Databases ${ }^{25}$ ): (a) Training image 1 (b) Training image 2 (c) Training image 3 (d) Test input image (e) Itti's saliency map (f) Itti's saliency map represented as a heat map (g) our saliency map (h) our saliency map represented as a heat map

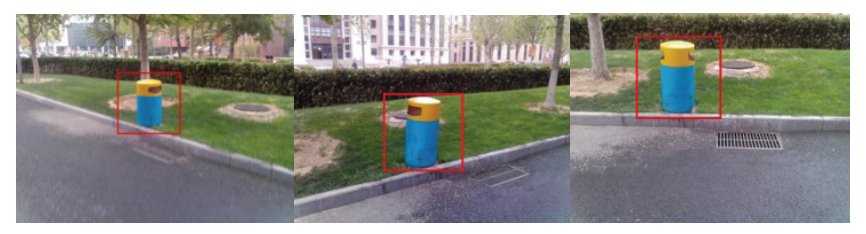

(a)

(b)

(c)

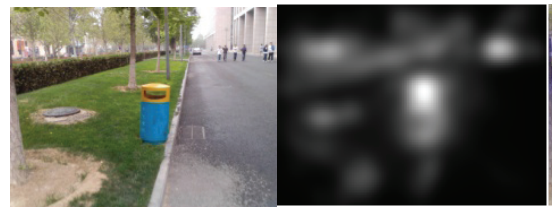

(d)

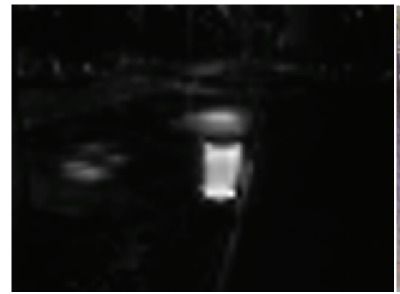

(g) (e)

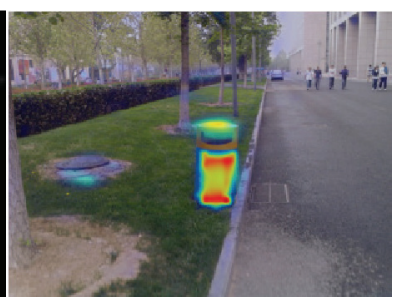

(h)
Fig. 6. Results of Experiment 3 where our target is a barrel (all images are taken in our campus): (a) Training image 1 (b) Training image 2 (c) Training image 3 (d) Test image (e) Itti's saliency map (f) Itti's saliency map represented as a heat map (g) our saliency map (h) our saliency map represented as a heat map

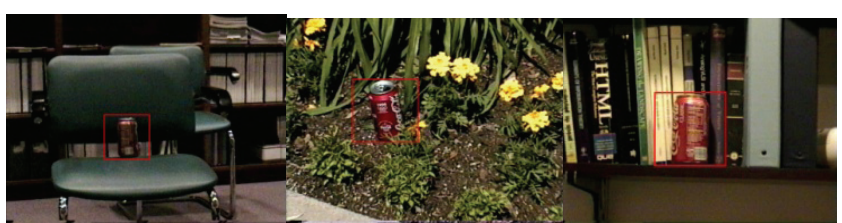

(a)

(b)

(c)

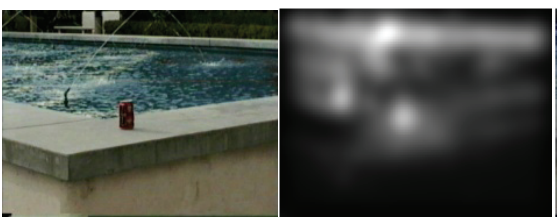

(d)

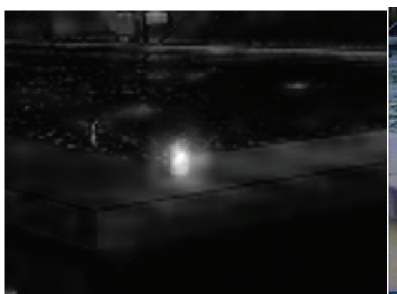

(g) (e)

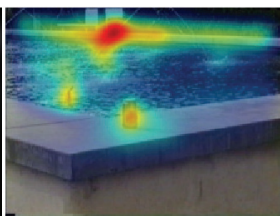

(f)

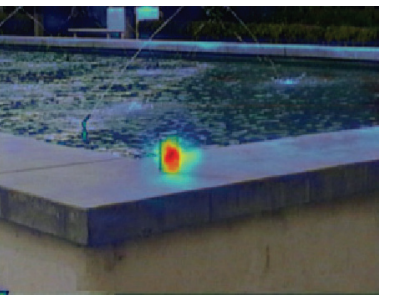

(h)
Fig. 5. Results of Experiment 2 where our target is a can (all images are from iLab Image Databases ${ }^{25}$ ): (a) Training image 1 (b) Training image 2 (c) Training image 3 (d) Test image (e) Itti's saliency map (f) Itti's saliency map represented as a heat map (g) our saliency map (h) our saliency map represented as a heat map

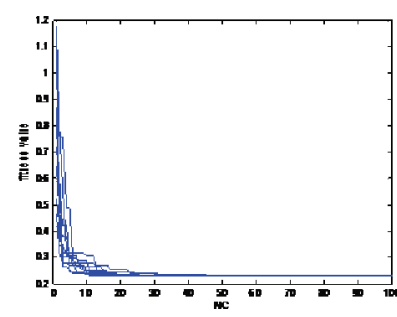

(a) CRO 10 times

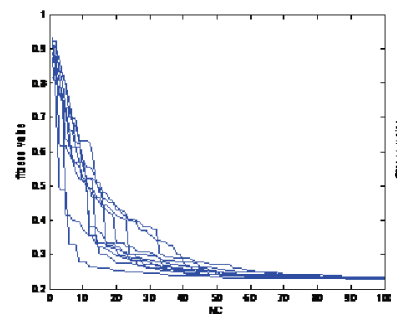

(c) BBO 10 times

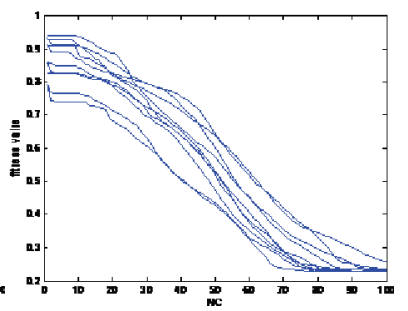

(b) PSO 10 times

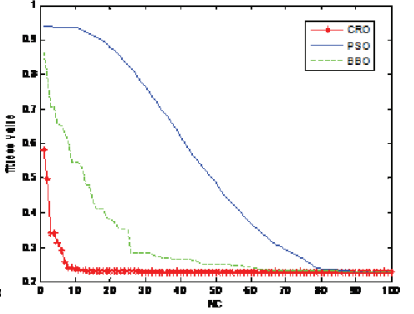

(d) CRO, PSO and BBO

Fig. 7. Evolution curves of CRO, PSO and BBO in our experiments. 
population size of the three algorithms. In this table, each result reported is an average value obtained by 50 independent runs. Compared with PSO and BBO, CRO has a much faster convergence speed, finding out the best solution at about $7 \mathrm{~s}$ on average. Therefore, a valid conclusion can be drawn that our CRO training procedure is not only effective but also efficient in visual attention.

\section{Conclusion}

This paper presented a CRO-based visual attention method to train weight coefficients with which feature maps are combined into a saliency map for a specific visual task. In our method, the weight coefficients are represented as molecular structure optimized by CRO algorithm. When CRO works out an optimum, the optimal weight coefficients and a saliency map used these coefficients with better performance will be obtained.

Comparative experiments have demonstrated the effectiveness of our training procedure to make the target more conspicuous than the background in a saliency map. Our task-driven training procedure makes the bio-inspired visual attention model more intelligent and more similar to the behavior of human in real life. As analyzed above, CRO has an extraordinarily fast convergence speed, which makes the training procedure very efficient.

In the future, we will make a systematic analysis of the way how weight coefficients inhibit image noise and highlight the target. Besides, integrating other top-down and bottom-up mechanisms to improve the performance of bio-inspired visual attention is another important direction for future work.

\section{Acknowledgements}

This work was partially supported by National Natural Science Foundation of China under grant \#61425008, \#61333004, \#61273054 and \#61175109, National Key Basic Research Program of China (973 Project) under grant \#2014CB046401 and \#2013CB035503, TopNotch Young Talents Program of China, Aeronautical Foundation of China under grant \#20135851042, and by the Graduate Innovation Foundation for Beihang University under Grant YCSJ-01-2014-01.

\section{References}

1. L. Laurent and C. Koch, A saliency-based search mechanism for overt and covert shifts of visual attention, Vision Research, 40(10-12) (2000) 1489-1506.

2. L. Itti, C. Koch and E. Niebur, A model of saliency-based visual attention for rapid scene analysis, IEEE Transactions on Pattern Analysis and Machine Intelligence, 20(11) (1998) 1254-1259.

3. D. Gao, S. Han and N. Vasconcelos, Discriminant saliency, the detection of suspicious coincidences, and applications to visual recognition, IEEE Trans. Pattern Analysis and Machine Intelligence, 31(6) (2009) 9891005 .

4. T. Liu, J. Sun, N. N. Zheng, X. Tang and H. Y. Shum, Learning to Detect A Salient Object, in Proc. IEEE Conference on Computer Vision and Pattern Recognition, (Minneapolis, Minnesota, 2007), pp. 1-8.

5. A. Borji and L. Itti, State-of-the-art in visual attention modeling, IEEE Trans. Pattern Analysis and Machine Intelligence, 35(1) (2013) 185-207.

6. N. Murray, M. Vanrell, X. Otazu and C. A. Parraga, lowlevel spatiochromatic grouping for saliency estimation, IEEE Transactions on Pattern Analysis and Machine Intelligence, 35(11) (2013) 2810-2816.

7. J. E. Raymond, K. L. Shapiro and K. M. Arnell, Temporary suppression of visual processing in an RSVP task: an attentional blink? Journal of Experimental Psychology, 18(3) (1992) 849-860.

8. D. J. Simons and C. F. Chabris, Gorillas in our midst: sustained inattentional blindness for dynamic events, Perception, 28(9) (1999) 1059-1074.

9. S. Kastner and L. G. Ungerleider, Mechanisms of visual attention in the human cortex, Annual Review of Neuroscience, 23 (2000) 315-341.

10. E.T. Rolls and G. Deco, Attention in natural scenes: neurophysiological and computational bases, Neural Networks, 19(9) (2006) 1383-1394.

11. L. Itti and C. Koch, Computational modelling of visual attention, Nature Reviews Neuroscience, 2(3) (2001) 194-203.

12. V. Navalpakkam and L. Itti, An integrated model of topdown and bottom-up attention for optimizing detection speed, in Proc. IEEE Conference on Computer Vision and Pattern Recognition, (New York, USA, 2006), pp. $2049-2056$.

13. L. Zhang, M. H. Tong, T. K. Marks, H. Shan and G. W. Cottrell, SUN: a Bayesian framework for saliency using natural statistics, Journal of Vision, 8(32) (2008) 1-20.

14. A. Y. S. Lam and V. O. K. Li, Chemical-reactioninspired metaheuristic for optimization, IEEE Transactions on Evolutionary Computation, 14(3) (2010) 381-399.

15. H. B. Duan and L. Gan, Elitist chemical reaction optimization for contour-based target recognition in aerial images, IEEE Transactions on Geoscience and Remote Sensing, 53(5) (2015) 2845-2859. 
16. H. B. Duan and L. Gan, Orthogonal multi-objective chemical reaction optimization for the brushless DC motor design, IEEE Transactions on Magnetics, 51(1) (2015) 7000207.

17. A. Y. S. Lam, J. Xu and V. O. K. Li, Chemical reaction optimization for population transition in peer-to-peer live streaming, in Proc. IEEE Congress on Evolutionary Computation, (Barcelona, Spain, 2010), pp. 1-8.

18. Y. Sun, A. Y. S. Lam, V. O. K. Li, J. Xu and J. J. Q. Yu, Chemical reaction optimization for the optimal power flow problem, in Proc. IEEE World Congress on Computational Intelligence, (Brisbane, Australia, 2012), pp. $1-8$.

19. C. Koch and S. Ullman, Shifts in selective visual attention: towards the underlying neural circuitry, Human Neurobiology, 4(4) (1985) 219-227.

20. A. Y. S. Lam and V. O. K. Li, Chemical reaction optimization: a tutorial, Memetic Computing, 4(1) (2012) 3-17.

21. H. B. Duan, Y. M. Deng, X. H. Wang and C. F. Xu, Small and dim target detection via lateral inhibition filtering and artificial bee colony based selective visual attention, PLoS ONE, 8(8) (2013) 1-12.

22. L. Gan and H. B. Duan, Biological image processing via chaotic differential search and lateral inhibition, OptikInternational Journal for Light and Electron Optics, 125(9) (2014) 2070-2075.

23. J. Kennedy and R. Eberhart, Particle swarm optimization, in Proc. IEEE International Conference on Neural Networks, (Perth, WA, 1995) 4 pp. 1942-1948.

24. X. H. Wang and H. B. Duan, Predator-prey biogeography-based optimization for bio-inspired visual attention, International Journal of Computational Intelligence Systems, 6(6) (2013) 1151-1162.

25. L. Itti and C. Koch, Feature combination strategies for saliency-based visual attention systems, Journal of Electronic Imaging, 10(1) (2001) 161-169. 